\title{
Influence of Reaction Parameters on the Hydrogenolysis of Hydroxymatairesinol Over Carbon Nanofibre Supported Palladium Catalysts
}

\author{
Heidi Bernas · Arie J. Plomp · Johannes H. Bitter • \\ Dmitry Yu. Murzin
}

Received: 28 April 2008/Accepted: 2 June 2008/Published online: 24 June 2008

(C) Springer Science+Business Media, LLC 2008

\begin{abstract}
The influence of catalyst particle size, stirring rate, catalyst mass $(0.2-0.6 \mathrm{~g})$, reaction temperature $\left(60-70{ }^{\circ} \mathrm{C}\right)$, and reactant concentration $(1.3-4 \mathrm{mmol} / \mathrm{L}$, with constant reactant/catalyst ratio) on the hydrogenolysis of the lignan hydroxymatairesinol (HMR) to matairesinol (MAT) was studied under hydrogen atmosphere using a carbon nanofibre supported palladium catalyst. When the temperature or HMR concentration was increased, the reaction rate increased as expected. However, the reaction rate was inversely dependent on the stirring rate, e.g., was influenced by external mass transfer, which was explained by the fact that the reaction rate decreases at higher hydrogen concentrations.
\end{abstract}

Keywords Lignan - Carbon nanofibres - Mass transfer . Kinetics

\section{Introduction}

Knots, e.g., the part of the branch that is embedded in the tree, are harmful in pulp and paper manufacturing and

H. Bernas · D. Yu. Murzin $(\bowtie)$

Laboratory of Industrial Chemistry and Reaction Engineering,

Process Chemistry Centre, Åbo Akademi University,

Biskopsgatan 8, 20500 Turku, Finland

e-mail: dmitry.murzin@abo.fi

H. Bernas

e-mail: heidi.bernas@abo.fi

\section{A. J. Plomp · J. H. Bitter}

Department of Inorganic Chemistry and Catalysis, Utrecht University, P.O. Box 80083, Utrecht, 3508 TB, The Netherlands

e-mail: a.j.plomp@uu.nl

J. H. Bitter

e-mail: j.h.bitter@uu.nl should be removed before pulping [1]. Some years ago, large quantities of the lignan hydroxymatairesinol (HMR) were discovered in Norway spruce (Picea abies) knots [2]. Since lignans have anticarcinogenic [3] and antioxidative [4] effects, the waste material from pulping industry can be used for synthesis of pharmaceuticals and technical antioxidants. No other lignan has been found in substantial amounts in nature, therefore other lignans that are of great interest and importance should be synthesized starting from HMR. The lignan matairesinol (MAT) has high application potential due to the fact that MAT was concluded to be superior to HMR in studies performed in vivo; more precisely the quantity of the mammalian lignan enterolactone, which reduces the cancer risk, was over twofold when MAT was used as precursor compared to HMR [5]. High radical and superoxide scavenging activities were also observed for MAT [6].

In our previous studies related to HMR hydrogenolysis, we have investigated the influence of support acidity for palladium catalysts supported on carbon [7], zeolites [8] and carbon nanofibres (CNF) [9]. For both carbon [7] as well as CNF [9] supported palladium catalysts, it was concluded that the activity increased when the amount of acid sites was increased. For Pd/CNF also the selectivity to MAT increased with acidity. On the other hand, in the case of palladium catalysts on supports with much stronger acid sites, i.e., H-Beta zeolites [8], the activity decreased as the concentration of acid sites increased. Palladium is required for the hydrogenolysis reaction, since the support itself without palladium did not give any MAT $[8,9]$. It was concluded that Brønsted acid sites are needed for the hydrogenolysis reaction; $\mathrm{Pd} / \mathrm{SiO}_{2}$ and $\mathrm{Pd} / \mathrm{Al}_{2} \mathrm{O}_{3}$ showed low activity in comparison to the zeolites used (alumina, in comparison to zeolites, has small amounts of Brønsted and high amount of Lewis acid sites, and silica has even lower 
amount of acid sites than alumina) [8]. The deactivation and solvent effects were studied in [10]. The solvents ethanol, 2-propanol, tert-butanol, 2-pentanol, as well as a mixture of 2-propanol and water $(50: 50 \mathrm{vol} / \mathrm{vol})$ were investigated. Ethanol, 2-propanol, and 2-pentanol gave the highest activity and the selectivity to MAT increased with the carbon chain length of the solvent (the highest selectivity was achieved in 2-pentanol). The activity was dramatically decreased as water was mixed with 2-propanol.

Since the choice of catalyst support and the concentration of acid sites on the support have a great influence on the hydrogenolysis to HMR, it is important to study the effect of reaction conditions. The influence of catalyst particle size, stirring rate, catalyst mass, temperature, and HMR concentration was studied. The experiments were done in a stirred glass reactor at atmospheric hydrogen pressure using a $1 \% \mathrm{Pd} / \mathrm{CNF}$ catalyst.

\section{Experimental}

The isolation of hydroxymatairesinol from Norway spruce knots is described in detail in [7]. There are two diastereomers of hydroxymatairesinol, $\left(7 R, 8 R, 8^{\prime} R\right)-(-)-7-$ allo-hydroxymatairesinol (HMR 1) and $\left(7 S, 8 R, 8^{\prime} R\right)-(-)-7-$ hydroxymatairesinol (HMR 2), the ratio between HMR 1 and HMR 2 can vary from tree to tree. The reaction scheme is illustrated in Fig. 1. The main reaction is hydrogenolysis of HMR to the desired product matairesinol (MAT), which can react to by-products A-D. Isomerization between HMR 1 and HMR 2 can take place. Moreover, HMR and the solvent 2-propanol react to 7-iso-propoxymatairesinol (7-i-propoxyMAT, two diastereomers).

A $1 \% \mathrm{Pd} / \mathrm{CNF}$ catalyst was utilized in the experiments. For growing of the $\mathrm{CNF}$, a Ni/SiO${ }_{2}$ catalyst was used. The synthesis of the $\mathrm{Ni} / \mathrm{SiO}_{2}$ growth catalyst and the growing of $\mathrm{CNF}$ are described in detail in [9]. The CNF were grown in a quartz vessel inside a tubular furnace at $550{ }^{\circ} \mathrm{C}$ for $24 \mathrm{~h}$ in a flow of $\mathrm{N}_{2}(480 \mathrm{~mL} / \mathrm{min}), \mathrm{H}_{2}(96 \mathrm{~mL} / \mathrm{min})$, and $\mathrm{CO}$ $(266 \mathrm{~mL} / \mathrm{min})$. The product was refluxed in $1 \mathrm{M}$ potassium hydroxide $(500 \mathrm{~mL})$ and, after filtration and washing, refluxed in concentrated nitric acid (Merck, 65\%) and then filtered. The residue was washed three times with deionised water and dried overnight at $120^{\circ} \mathrm{C}$.

The deposition of palladium on CNF was performed via an ion adsorption method according to [11]. The oxidised $\mathrm{CNF}(25.00 \mathrm{~g})$ were suspended in de-ionised water $(100 \mathrm{~mL})$. The $\mathrm{pH}$ of the suspension was adjusted at a value between 5 and 6 by adding ammonium hydroxide solution (Acros, 25\%). Subsequently, $5.0 \mathrm{~g}$ of $\mathrm{Pd}\left(\mathrm{NH}_{3}\right)_{4}\left(\mathrm{NO}_{3}\right)_{2}$-solution (Johnson Matthey; 5.04 wt.\% Pd) was added and the resulting suspension was stirred for $20 \mathrm{~h}$ at room temperature under nitrogen atmosphere. The

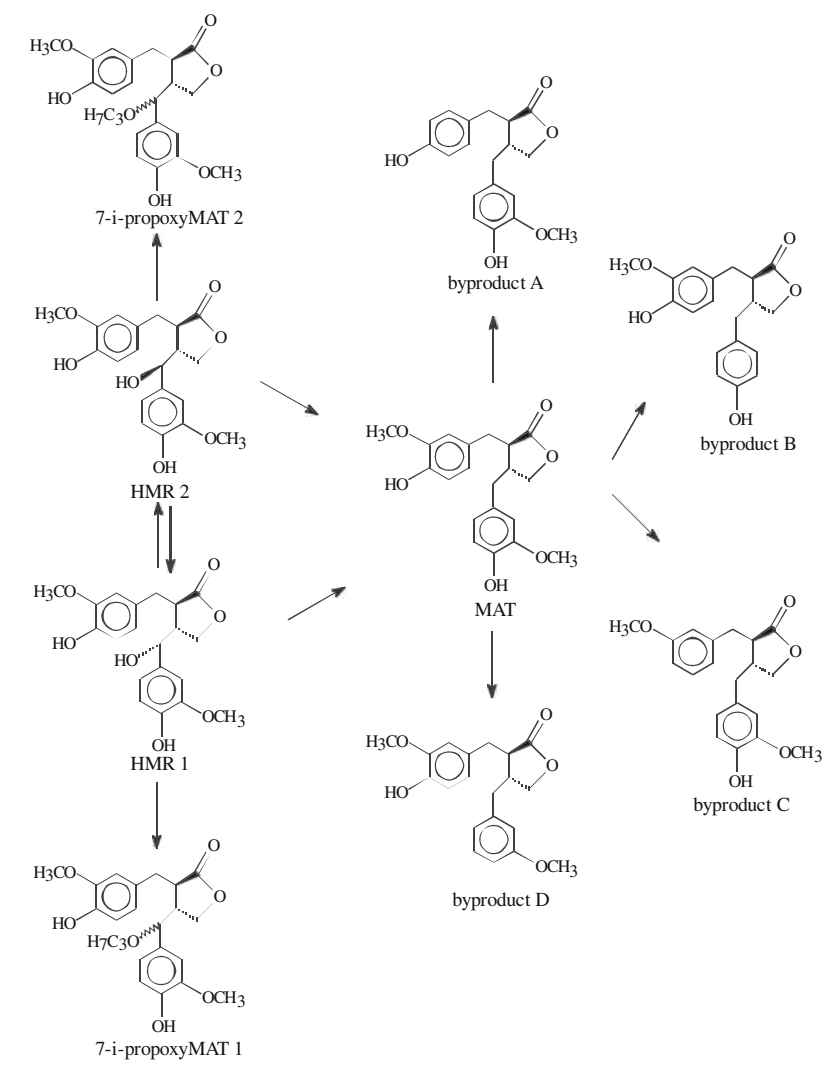

Fig. 1 Reaction scheme for hydrogenolysis of hydroxymatairesinol (HMR) to matairesinol (MAT), etherification of HMR to 7-isopropoxymatairesinol (7-i-propoxyMAT), and hydrogenolysis of MAT to byproducts $\mathrm{A}-\mathrm{D}$

catalyst was filtered under nitrogen flow, washed and dried at $80{ }^{\circ} \mathrm{C}$ for $20 \mathrm{~h}\left(\right.$ ramp $\left.5{ }^{\circ} \mathrm{C} / \mathrm{min}\right)$ under nitrogen flow. The $\mathrm{Pd} / \mathrm{CNF}$ catalyst was reduced in hydrogen flow $(96 \mathrm{~mL} / \mathrm{min})$ and nitrogen flow $(480 \mathrm{~mL} / \mathrm{min})$ at $250{ }^{\circ} \mathrm{C}$ (ramp $5{ }^{\circ} \mathrm{C} / \mathrm{min}$ ) and 1.33 bar for $2 \mathrm{~h}$. The obtained $\mathrm{Pd} /$ CNF is denoted Pd/CNF-ox.

The experiments were done in a $200 \mathrm{~mL}$ glass reactor equipped with a heating jacket, a re-flux condenser, oil lock, pitched-blade turbine, and stirring baffles. The Pd/CNF-ox catalyst was pre-activated in-situ in a hydrogen (AGA, $99.999 \%)$ flow $(100 \mathrm{~mL} / \mathrm{min})$ at $100{ }^{\circ} \mathrm{C}$ for $1 \mathrm{~h}$, thereafter the reactor was cooled down to the reaction temperature in a nitrogen (AGA, 99.999\%) flow (100 $\mathrm{mL} / \mathrm{min})$. The reactant solution, consisting of hydroxymatairesinol (HMR2/HMR1 initial ratio $=3.6$ ) dissolved in $100 \mathrm{~mL}$ 2-propanol, was deoxygenated by nitrogen flow $(100 \mathrm{~mL} / \mathrm{min})$ for $10 \mathrm{~min}$ in a glass tube. After pouring the reactant solution into the reactor, the stirring was started at reaction time set to zero, the gas flow was changed to hydrogen $(100 \mathrm{~mL} / \mathrm{min})$ and the first sample was withdrawn.

Samples were withdrawn at different time intervals and analysed by a gas chromatograph (GC) equipped with a HP-1 column (length $25 \mathrm{~m}$, inner diameter $0.20 \mathrm{~mm}$, film 
thickness $0.11 \mu \mathrm{m}$ ) and a flame ionisation detector (FID) operating at $300{ }^{\circ} \mathrm{C}$. The samples were silylated prior to analysis using $N, O$-bis(trimethylsilyl)trifluoro-acetamide. The silylation procedure and the analysis are described more in detail in [9].

\section{Results and Discussion}

\subsection{Mass Transfer}

It is well known that, if the diffusion of reactants and products is fast compared with the reaction steps on the surface, the overall reaction rate is not dependent on the diffusion steps [12]. On the other hand, if the surface reactions are very fast compared with the diffusion steps, the overall reaction rate is affected by the mass transport. If the diffusion of the bulk gas or liquid to the catalyst surface affects the rate, a change in the flow conditions would influence the overall rate.

The dependence of the mass transfer coefficient and the flow conditions is visible through various correlations between dimensionless numbers routinely applied to calculate the impact of film (gas/liquid) and external diffusion $[13,14]$. One of such correlations [14] gives the following equation for the liquid-solid mass transfer

$\beta_{\mathrm{LS}}=\left(\frac{\varepsilon D^{4} \rho}{\varepsilon d_{\mathrm{p}}^{2}}\right)^{1 / 6}$

where $\varepsilon$ denotes the specific mixing power, $D$ is the diffusion coefficient of solute in a solvent, $\rho$ and $\eta$ are, respectively, solvent density and solvent viscosity, $d_{\mathrm{p}}$ is the diameter of the catalyst particles. The energy dissipation is a function of mixing power $W$, often related to the stirring speed according to

$\varepsilon=\frac{W}{m}=K \rho_{\text {liquid }} n^{3} d_{\text {(stirrer) }}^{5} / m$

where $n$ is the stirring speed, $m$ is the mass of the liquid, $K$ is a constant specific for a particular impeller and $d_{\text {stirrer }}$ is the stirrer diameter. The dependence of the mass transfer coefficient on stirring speed can be thus established.

$\beta \propto n^{1 / 2}$

If the rate is then independent on the agitation efficiency at sufficiently high stirring speed, then it is conventionally assumed, that mass transport effects are minimized.

The impact of internal diffusion is usually assessed through calculations of the catalyst effectiveness factor, which depends only the Thiele modulus for an isothermal catalyst grain $\eta_{\mathrm{e}}=f\left[\varphi_{s},\right]$. The Thiele modulus for instance of a first-order reaction is defined as
$\varphi_{s}=\frac{V_{\mathrm{p}}}{A_{\mathrm{p}}} \sqrt{\frac{k}{D_{\mathrm{ei}}}}$

where $A_{\mathrm{p}}$ and $V_{\mathrm{p}}$ are, respectively, surface area and volume of a catalyst particle, $k$ is the rate constant and $D_{\mathrm{e}}$ is the effective diffusion coefficient.

A change in the flow conditions (e.g., specific mixing power) does not affect the mass transport in the catalyst pores directly, meaning that the overall rate could still be limited by internal diffusion but unaffected by external flow conditions. It can happen if catalyst particles are large enough to have an impact on internal diffusion (i.e., Thiele modulus) but the stirring is very efficient to overcompensate the potential negative influence of catalyst particle size increase on mass transfer (Eq. 1). Alternatively the overall rate can be retarded by external, but not influenced by internal diffusion (small catalyst particles and inefficient stirring). In the present study, the influence of mass transfer was investigated and the effect of catalyst particle size (internal mass transfer in the pores of the catalyst), the stirring rate (external mass transfer from bulk to surface) and the catalyst mass (gas-to-liquid mass transfer) was studied.

Figure 2a shows the conversion versus time as the catalyst particle size is varied. The lowest reaction rate was obtained for the largest catalyst particles, 90-125 $\mu \mathrm{m}$, and the reaction rate increased when the catalyst particle size decreased to $45-90 \mu \mathrm{m}$. As an experiment using particles $<125 \mu \mathrm{m}$ was made, where $75 \%$ of the particles were smaller than $90 \mu \mathrm{m}$, the reaction rate for this experiment was the same as for the experiment where particles in the interval $45-90 \mu \mathrm{m}$ were used. The selectivity to MAT (Fig. 2b) was approximately the same for all fractions used.

It is visible from Fig. 3 that the conversion as a function of time increased when the stirring rate decreased from 1000 to $600 \mathrm{rpm}$. If the overall reaction rate is not affected by the change in stirring rate, it is usually concluded that external mass transfer is not limiting the overall rate. Note that the decrease in stirring rate had a positive effect on the reaction rate, which is not conventional. This is explained by the fact that hydrogenolysis of HMR displays a rather unusual dependence of the concentration of hydrogen, in particular negative reaction order in hydrogen pressure [15]. Hydrogen is needed for the hydrogenolysis of HMR to take place, since experiments under nitrogen atmosphere gave the dehydrogenation product oxomatairesinol [8]. Too much hydrogen retards the hydrogenolysis reaction and this behaviour would explain why the reaction rate is faster at lower stirring rate, when the transfer of hydrogen from the liquid to the catalyst surface is retarded.

The influence of gas-to-liquid mass transfer was investigated by varying the catalyst mass. When the conversion 

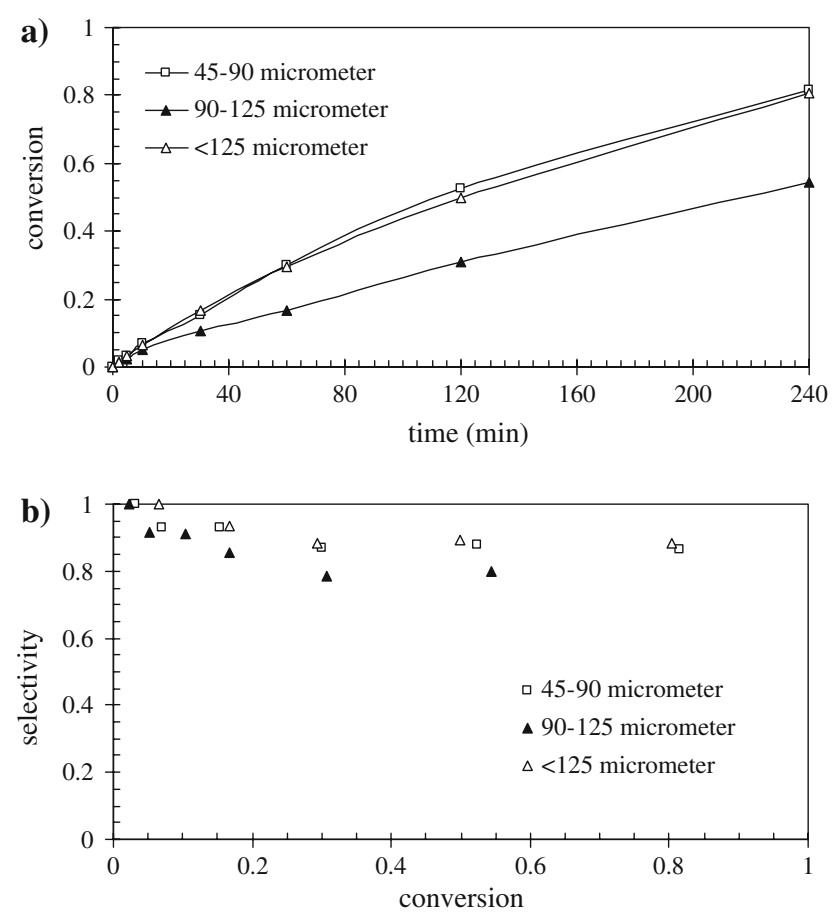

Fig. 2 Hydrogenolysis of HMR to MAT, influence of catalyst particle size: (a) conversion versus time, (b) selectivity to MAT versus conversion. Conditions: $0.4 \mathrm{~g}$ catalyst, $2.7 \mathrm{mmol} / \mathrm{L}$ HMR in 2-propanol, $70{ }^{\circ} \mathrm{C}$ reaction temperature, HMR2/HMR 1 ratio $=3.6$, stirring rate $1000 \mathrm{rpm}$. Fraction $<125 \mu \mathrm{m}$ consists of $75 \mathrm{wt} . \%<90 \mu \mathrm{m}$ and 25 wt-\% 90-125 $\mu \mathrm{m}$

as a function of the normalised abscissa time multiplied by catalyst mass is plotted (Fig. 4a), the kinetic curves should coincide if gas-to-liquid mass transfer is absent. As 0.2 and $0.4 \mathrm{~g}$ catalyst were used, the curves overlap, while with $0.6 \mathrm{~g}$ of catalyst the conversion was around $10 \%$ higher. Under gas-to-liquid mass transfer conditions higher mass gives a lower conversion, however, in the present case an opposite behaviour was obtained, which nevertheless can be explained by the presence of hydrogen-to-liquid mass

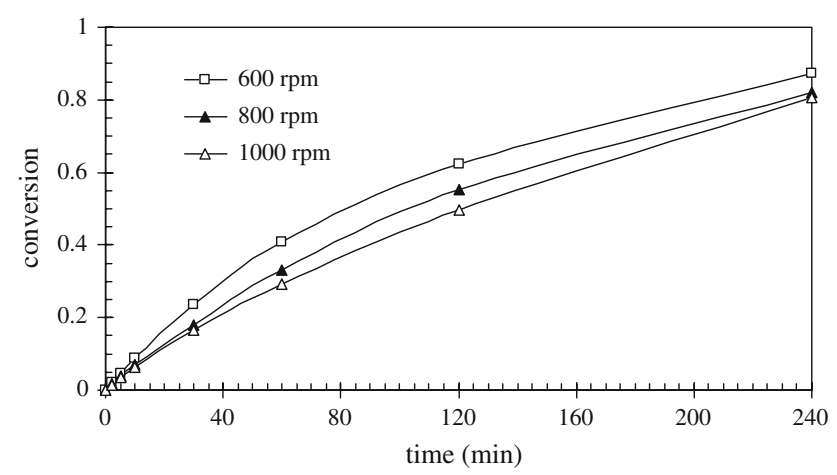

Fig. 3 Hydrogenolysis of HMR to MAT, influence of stirring rate, conversion versus time. Conditions: $0.4 \mathrm{~g}$ catalyst (catalyst particle size $<125 \mu \mathrm{m}$ ), $2.7 \mathrm{mmol} / \mathrm{L} \mathrm{HMR}$ in 2-propanol, $70{ }^{\circ} \mathrm{C}$ reaction temperature, HMR2/HMR1 ratio $=3.6$ transfer at higher catalyst mass, which favours the hydrogenolysis reaction, as too much hydrogen would retard the reaction. Figure $4 \mathrm{~b}$ shows the selectivity to MAT versus conversion; the selectivity was around $90 \%$ for all experiments. It is visible from Fig. $4 \mathrm{c}$ that the HMR2-toHMR1 ratio decreased from 3.6 at the beginning of the reaction to 0.5 at almost full conversion.

\subsection{Effect of Temperature}

The effect of reaction temperature was studied in the temperature interval $60-70{ }^{\circ} \mathrm{C}$ and the conversion versus time is shown in Fig. 5a and the selectivity to MAT versus
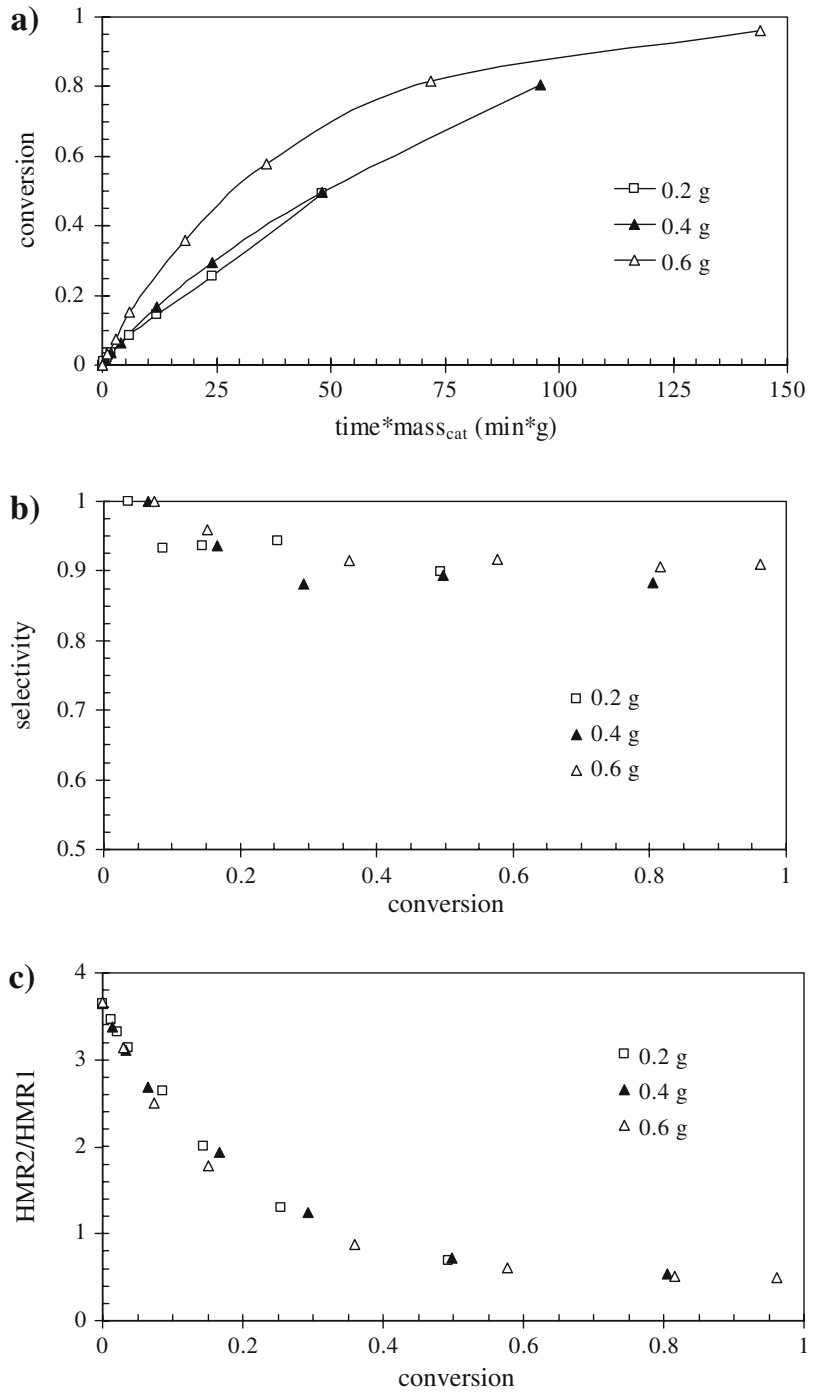

Fig. 4 Hydrogenolysis of HMR to MAT, influence of catalyst mass: (a) conversion versus time multiplied by catalyst mass, (b) selectivity to MAT versus conversion, and (c) HMR2-to-HMR1 ratio versus conversion. Conditions: $2.7 \mathrm{mmol} / \mathrm{L} \mathrm{HMR}$ in 2-propanol, catalyst particle size $<125 \mu \mathrm{m}, 70{ }^{\circ} \mathrm{C}$ reaction temperature, HMR2/HMR1 initial ratio $=3.6$, stirring rate $1000 \mathrm{rpm}$ 

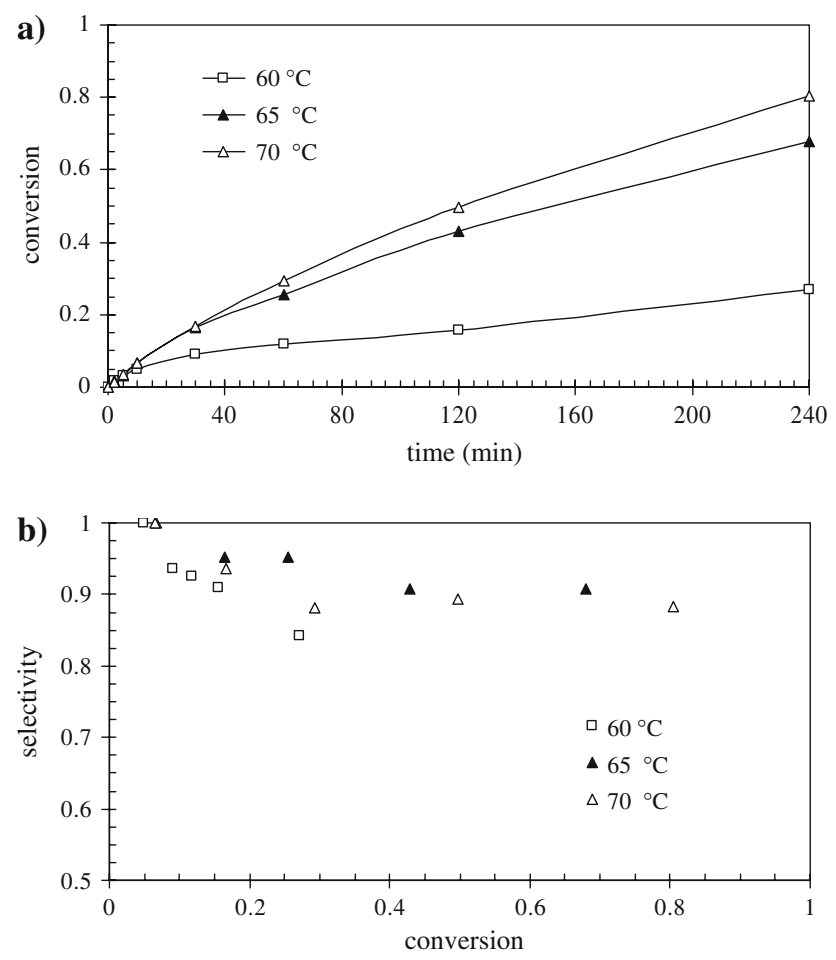

Fig. 5 Hydrogenolysis of HMR to MAT, influence of reaction temperature: (a) conversion versus time, (b) selectivity to MAT versus conversion. Conditions: $0.4 \mathrm{~g}$ catalyst, catalyst particle size $<125 \mu \mathrm{m}, 2.7 \mathrm{mmol} / \mathrm{L}$ HMR in 2-propanol, HMR2/HMR1 initial ratio $=3.6$, stirring rate $1000 \mathrm{rpm}$

conversion in Fig. 5b. The reaction rate increased with the temperature (Fig. 5a), but the difference in reaction rate between 60 and $65^{\circ} \mathrm{C}$ was much larger than between 65 and $70^{\circ} \mathrm{C}$. Since the reaction rate at $60{ }^{\circ} \mathrm{C}$ was low, temperatures below $60{ }^{\circ} \mathrm{C}$ were not used. Neither were temperatures higher than $70{ }^{\circ} \mathrm{C}$ utilized, this limitation was imposed by the boiling point of the solvent 2-propanol. The selectivity to MAT (Fig. 5b) was slightly lower at $60{ }^{\circ} \mathrm{C}$ compared to other temperatures.

\subsection{Effect of Reactant and Hydrogen Concentration from Mechanistic Point of View}

The HMR/catalyst ratio was kept constant as the influence of HMR concentration was studied. Figure 6 shows conversion as a function of time and selectivity to MAT versus conversion. Both reaction rate and selectivity to MAT increased with increase of HMR concentration, which means that selectivity to propoxyMAT increased when the concentration of HMR and catalyst decreased. The increased propoxyMAT selectivity cannot be attributed to an increased 2-propanol-to-HMR ratio, since the 2-propanol is in large excess. As already mentioned above for the influence of catalyst mass, hydrogen-to-liquid mass transfer is present at higher catalyst mass meaning that there is
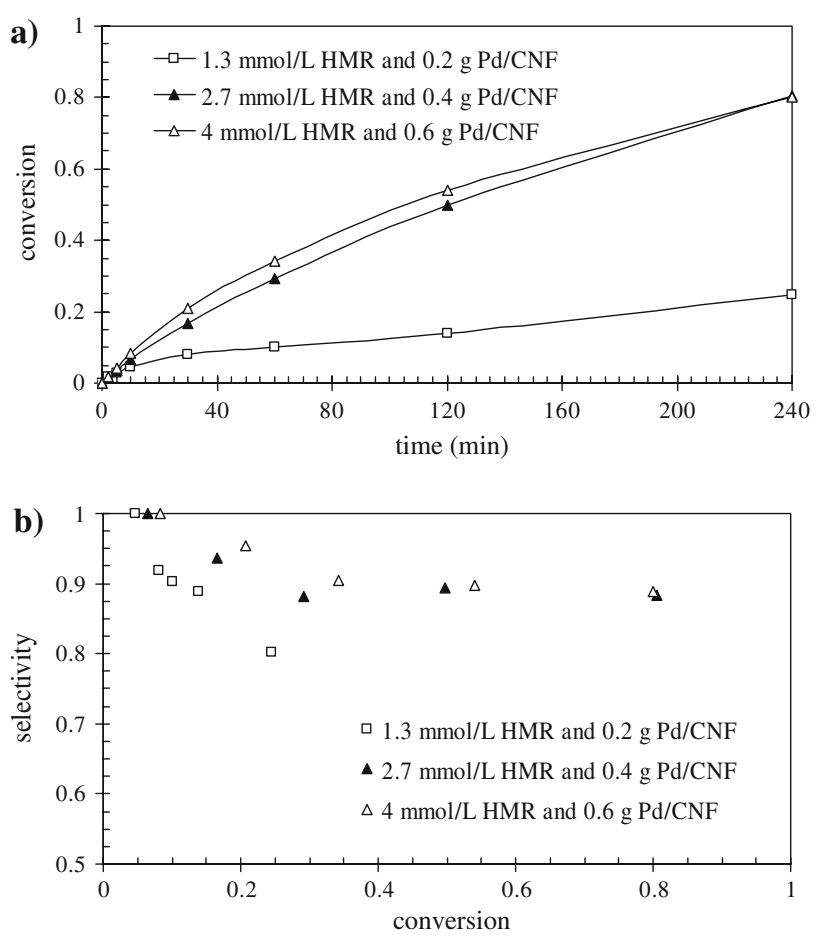

Fig. 6 Hydrogenolysis of HMR to MAT, influence of concentration: (a) conversion versus time, (b) selectivity to MAT versus conversion. Conditions: 2-propanol as solvent, catalyst particle size $<125 \mu \mathrm{m}$, $70{ }^{\circ} \mathrm{C}$ reaction temperature, HMR2/HMR 1 initial ratio $=3.6$, stirring rate $1000 \mathrm{rpm}$

more hydrogen on the catalyst surface for a lower catalyst mass. More hydrogen on the surface has a negative effect on the hydrogenolysis of HMR to MAT, whereas the etherification of HMR to propoxyMAT is not affected since the etherification also takes place when a CNF without metal is used [9].

Experimental data presented above point out reaction kinetics with negative order in hydrogen. Kinetic regularities adhering to negative order dependences are typically explained by so-called Langmuir-Hinshelwood kinetics, when besides a competition for adsorbed sites on the surface there is also a nonlinear elementary step. The latter means that the rate expression contains two terms, representing coverage of reactive species. In hydrogenation reactions with such competition between bulky organic molecules and hydrogen, negative pressure dependence in hydrogen is not observed and at high pressures the reaction kinetics usually follows zero-order in hydrogen. On the other hand, in hydrogenolysis reactions and some catalytic transformations of hydrocarbons, e.g., hydrocarbons isomerization, negative reaction orders were observed [1619]. Experimental data on methylpentane transformations on Pt/zeolite catalysts were explained invoking a mechanism requiring several surface sites for adsorption of the substrate [16]. Moreover, when adsorption of the reactant 
occurs on a cluster of sites, presence of adsorbed hydrogen is required for the adsorption to proceed. In essence it means that there is a following step in the reaction mechanism

$\mathrm{MAT}-\mathrm{OH}+\mathrm{Z}^{*}+\mathrm{H}^{*}=\mathrm{MATZ}^{*}+\mathrm{H}_{2} \mathrm{O}+*$

where MAT-OH is equal to HMR, $\mathrm{Z}$ is the number of potential free sites needed for adsorption of a bulky organic molecule, $\mathrm{H}^{*}$ stands for adsorbed hydrogen, which is generated through hydrogen chemisorption

$\left(\mathrm{H}_{2}+2^{*}=2 \mathrm{H}^{*}\right)$.

High pressure of hydrogen will eventually lead to high hydrogen coverage and in such way will prevent adsorption of HMR displaying negative order in hydrogen pressure. However, detailed kinetic analysis of experimental data on HMR hydrogenolysis is required and will be a subject of a separate paper.

\section{Conclusions}

The natural lignan hydroxymatairesinol (HMR) was hydrogenolysed to matairesinol (MAT), which has a potential to be used in pharmaceuticals, as a food additive or as a technical antioxidant. The reaction kinetics was studied by varying the catalyst particle size, stirring rate, catalyst mass $(0.2-0.6 \mathrm{~g})$, reaction temperature $\left(60-70{ }^{\circ} \mathrm{C}\right)$, and HMR concentration (1.3-4 mmol/L, constant $\mathrm{HMR} /$ catalyst ratio). The kinetic experiments were done under hydrogen atmosphere in a stirred glass reactor using a CNF supported palladium $(1 \% \mathrm{Pd} / \mathrm{CNF})$ catalyst.

The reaction rate increased when the stirring rate decreased. Since the rate of mass transfer from the bulk to the catalyst surface decreases when the stirring rate decreases, the increase in reaction rate is explained by the decrease in adsorbed hydrogen and a subsequent increase in the reaction rate; too much hydrogen on the catalyst surface is retarding the hydrogenolysis of HMR. A positive effect of gas-to-liquid mass transfer was noticed when the catalyst mass was varied being consistent with negative reaction order in hydrogen. As expected, the reaction rate increased when the temperature was increased. The reaction rate as well as the selectivity to MAT increased with increasing HMR concentration at constant HMR-to-catalyst ratio.
Acknowledgments This work is part of the activities at the Ábo Akademi Process Chemistry Centre within the Finnish Centre of Excellence Programme (2000-2011) by the Academy of Finland. Financial support from the European Union through the Sixth Framework Programme (Project 506621) is gratefully acknowledged. The authors express their gratitude to Mr. Christer Eckerman for the HMR preparation.

\section{References}

1. Holmbom B, Eckerman C, Eklund P, Hemming J, Nisula L, Reunanen M, Sjöholm R, Sundberg A, Sundberg K, Willför S (2003) Phytochem Rev 2:331

2. Willför S, Hemming J, Reunanen M, Eckerman C, Holmbom B (2003) Holzforschung 57:27

3. Adlercreutz H (2002) Lancet 3:364

4. Willför SM, Ahotupa MO, Hemming JE, Reunanen MHT, Eklund PC, Sjöholm RE, Eckerman CSE, Pohjamo SP, Holmbom BR (2003) J Agric Food Chem 51:7600

5. Saarinen NM, Smeds A, Mäkelä SI, Ämmälä J, Hakala K, Pihlava J-M, Ryhänen E-L, Sjöholm R, Santti R (2002) J Chrom B $777: 311$

6. Yamauchi S, Sugahara T, Nakashima Y, Okada A, Akiyama K, Kishida T, Maruyama M, Masuda T (2006) Biosci Biotechnol Biochem 70:1934

7. Markus H, Mäki-Arvela P, Kumar N, Kul'kova NV, Eklund P, Sjöholm R, Holmbom B, Salmi T, Murzin DY (2005) Catal Lett 103:125

8. Markus H, Mäki-Arvela P, Kumar N, Heikkilä T, Lehto V-P, Sjöholm R, Holmbom B, Salmi T, Murzin DY (2006) J Catal 238:301

9. Markus H, Plomp AJ, Mäki-Arvela P, Bitter JH, Murzin DY (2007) Catal Lett 113:141

10. Markus H, Mäki-Arvela P, Kumar N, Salmi T, Murzin DY (2007) Catal Today 121:100

11. Winter F, van Dillen AJ, de Jong KP (2004) J Mol Catal A 219:273

12. Fogler HS (1999) Elements of chemical reaction engineering. Prentice-Hall International Series in the Physical and Chemical Engineering Sciences, New Jersey

13. van Santen RA, van Leeuwen PWNM, Moulijn AA, van Averill BA (2002) Catalysis: an integrated approach. Elsevier, Amsterdam

14. Murzin DY, Salmi T (2005) Catalytic kinetics. Elsevier, Amsterdam, p 492

15. Markus H, Mäki-Arvela P, Holmbom B, Salmi T, Murzin DY (2005) In: Levec J, Pintar A (eds) Book of extended abstracts for the 5th international symposium on catalysis in multiphase reactors and 4th international symposium on multifunctional reactors, Slovenia, pp 259-260

16. Siffert S, Murzin DY, Garin F (1999) Appl Catal A 178:85

17. Gault F (1981) Adv Catal 30:1

18. Frennet A, Lienard G, Crocq A, Degols L (1978) J Catal 53:150

19. Frennet A (1992) Catal Today 12:131 